\title{
UNILATERAL REGULATION BREAKS REGULARITY OF TURING PATTERNS
}

\author{
TOMÁŠ VEJCHODSKÝ, FILIP JAROŠ, MILAN KUČERA, VOJTĚCH RYBÁŘ
}

\begin{abstract}
We consider a reaction-diffusion system undergoing Turing instability and augment it by an additional unilateral source term. We investigate its influence on the Turing instability and on the character of resulting patterns. The nonsmooth positively homogeneous unilateral term $\tau v^{-}$has favourable properties, but the standard linear stability analysis cannot be performed. We illustrate the importance of the nonsmoothness by a numerical case study, which shows that the Turing instability can considerably change if we replace this term by its arbitrarily precise smooth approximation. However, the nonsmooth unilateral term and all its approximations yield qualitatively similar patterns although not necessarily developing from small disturbances of the spatially homogeneous steady state. Further, we show that the unilateral source breaks the approximate symmetry and regularity of the classical patterns and yields asymmetric and irregular patterns. Moreover, a given system with a unilateral source produces spatial patterns even for diffusion parameters with ratios closer to 1 than the same system without any unilateral term.
\end{abstract}

\section{INTRODUCTION}

Reaction-diffusion systems are frequently used to model the initiation of animal forms and patterns. After publication of Turing's purely theoretical paper 38, growing number of biologists succeeded in matching empirical data with mathematical simulations. Morphogens with Turing-like behaviour were found in the process of hair follicles formation [31], the generation of transverse ridges of the palate [11] or patterning the germ layers [8, 36]. The concept of reactions and diffusion of morphogens was widened to the interactions of pigment cells. In the case of zebrafish, the validity of this model was tested on individuals with ablated skin [25, 26]. Turing's mechanism is also used to model the formation of coat patterns in mammals, see for example [30, 32.

We will introduce a unilateral term $\hat{g}(v)$ to the concrete reaction-diffusion system introduced in [4] to model skin patterns in fish and used in [30] to model coat patterns of jaguar and leopard, see (3). This particular system will be provided

1991 Mathematics Subject Classification. 35Q92, 92B05.

Key words and phrases. Reaction-diffusion system, diffusion driven instability, spatial patterns, unilateral source, irregular patterns, mutant colouration, king cheetah.

Date: 22 August 2017.

Published in Physical Review E 96, 022212 (2017), DOI: 10.1103/PhysRevE.96.022212. 
in Section 2 and it fits into a general scheme

$$
\begin{aligned}
& \frac{\partial u}{\partial t}=d_{1} \Delta u+f(u, v) \quad \text { in } \Omega, \\
& \frac{\partial v}{\partial t}=d_{2} \Delta v+g(u, v)+\hat{g}(v) \quad \text { in } \Omega
\end{aligned}
$$

with the usual homogeneous Neumann boundary conditions

$$
\frac{\partial u}{\partial n}=\frac{\partial v}{\partial n}=0 \quad \text { on } \partial \Omega .
$$

This system models the diffusion and nonlinear interactions of two morphogens. The domain $\Omega \subset \mathbb{R}^{2}$ represents the tissue, $t$ denotes the time variable, $d_{1}$ and $d_{2}$ are diffusion coefficients, smooth functions $f(u, v)$ and $g(u, v)$ describe interactions between the morphogens, and $n$ stands for the unit outward facing normal vector to the boundary $\partial \Omega$. In accordance with the original publications [4, 30], the quantities $u, v$ denote deviations of morphogens concentrations $U, V$ from a positive spatially homogeneous equilibrium concentrations $\bar{U}, \bar{V}$. Thus, negative deviations $u=U-\bar{U}$ and $v=V-\bar{V}$ can still correspond to positive concentrations $U$ and $V$. Values of $\bar{U}$ and $\bar{V}$ are not specified even in the original publications [4, 30]. In fact, they can be chosen arbitrarily and substitution $u=U-\bar{U}$ and $v=V-\bar{V}$ into (1) yields a system for concentrations $U$ and $V$. In all numerical calculations presented in this paper the deviations $u$ and $v$ lie within the range $(-1.5,1.5)$. Hence, if $\bar{U}>1.5, \bar{V}>1.5$ then concentrations $U=u+\bar{U}, V=v+\bar{V}$ remain positive.

A novelty in system (1) is the additional term $\hat{g}(v)$ which is unilateral in the sense that there is a threshold value $\theta$ such that

$$
\hat{g}(v)>0 \text { for } v<\theta \text { and } \hat{g}(v)=0 \text { otherwise. }
$$

This term describes an additional source active only if the concentration of the second morphogen decreases below the threshold $\theta$. The key point is that the function $\hat{g}(v)$ can be nonsmooth, a typical example being $\hat{g}(v)=\tau(v-\theta)^{-}$, where $(v-\theta)^{-}=(|v-\theta|-v+\theta) / 2$ stands for the negative part of $v-\theta$ and $\tau>0$ controls the strength of this unilateral source. An alternative example is the saturation term $\hat{g}(v)=\tau\left(1-\exp \left[-(v-\theta)^{-}\right]\right)$that models the limited ability of cells to produce morphogens.

We will have $f(0,0)=g(0,0)=0$. Thus, if $\hat{g}(0)=0$ then $(u, v)=(0,0)$ will be a constant stationary solution of (1) with (2). We will also consider cases when $\hat{g}(0) \neq 0$ and there exist nontrivial $(\bar{u}, \bar{v})$ satisfying $f(\bar{u}, \bar{v})=g(\bar{u}, \bar{v})+\hat{g}(\bar{v})=0$. Then $u=\bar{u}, v=\bar{v}$ is a constant stationary solution of (1) with (2). In both cases, we refer to this constant steady state $(\bar{u}, \bar{v})$ as a ground state. We say that system (1) with (2) undergoes the Turing diffusion driven instability if the ground state is stable with respect to small spatially homogeneous perturbations and unstable with respect to small spatially nonhomogeneous perturbations. Our goal will be to investigate the influence of the unilateral term $\hat{g}(v)$ on the Turing 
instability and on the formation of spatial patterns (spatially nonconstant stationary solutions). From these points of view we will compare the unilateral and classical systems, i.e. a system with a unilateral term $\hat{g}(v)$ and the corresponding classical system with $\hat{g} \equiv 0$.

In the classical (smooth) case, we can perform the well known linear analysis to find necessary conditions for the Turing instability to occur, see e.g. [12, 20, 32]. If these conditions are satisfied then starting from small nonhomogeneous disturbances of the ground state, the solution of (1)-(2) can converge to another, spatially nonhomogeneous steady state, provided it exists. In biology, this process of forming nonhomogeneous steady states can serve as a model of pattern (prepattern) formation mechanisms. Therefore, we often refer to these spatially nonhomogeneous stationary solutions as patterns. We will have $\partial f / \partial u(\bar{u}, \bar{v})>0$ in the system under consideration and we will call $u$ the activator. In this case, one of the necessary conditions for the Turing instability is that the diffusion coefficient of the activator $u$ is sufficiently smaller than the diffusion coefficient of $v$, i.e. the ratio $d_{1} / d_{2}$ is sufficiently small.

Our paper is motivated mainly by two surprising results [27] and [23] about systems in the form (1) with $\hat{g} \equiv 0$, where $f$ and $g$ satisfy assumptions under which Turing instability occurs. In these papers, a unilateral source is not given by the term $\hat{g}$ as in (1), but by certain unilateral conditions for $v$ formulated by variational inequalities. The former result guarantees existence of stationary spatially nonhomogeneous solutions even for $d_{1} / d_{2}$ arbitrarily large. The later result concerns certain instability of the ground state for a very wide range of values $d_{1}$ and $d_{2}$. There are also earlier theoretical studies, e.g. [10, 13, 14, 15, 29] and references therein, predicting new and interesting features of systems with various unilateral conditions or terms. Let us note that the original necessary condition of one fast and one slow diffusion can be removed or relaxed also by other approaches. For example, Turing instability can occur in the presence of cross-diffusion even if the (isolated) activator diffuses faster than the (isolated) inhibitor [16]. The presence of non-diffusing species yields patterns outside the classical limits of the Turing model [7, 24]. Both stochastic effects and crossdiffusive terms were shown [5] to yield stochastic self-organization for a wider region of parameters than in the conventional Turing approach. Similarly, stochastic patterns for a wider region of parameters were observed and analysed in [3] for the stochastic Brusselator model. Alternatively, nonnormality of the linear stability matrix can amplify the noise and yield fluctuation-induced Turing patterns that are not strongly limited by the value of diffusion coefficients [6].

Going back to unilateral regulation, the unilateral conditions described by variational inequalities considered in [23, 27] correspond to sources which do not allow $v$ to decrease below zero (i.e. the concentration $V$ is not allowed to decrease below the equilibrium concentration $\bar{V}$ ) on a given subset of the boundary or of the interior of the domain. These hard inequalities, however, seem to be unrealistic from the viewpoint of biological applications, because it is difficult to imagine 
a natural mechanism which would strictly prevent the concentration of a morphogen to decrease below the threshold. Therefore we consider a unilateral term $\hat{g}(v)$, which seems to be more realistic. It does not prevent $v$ to decrease below $\theta$, but it works against this decrease.

Our goal is to investigate this case and to find values of the ratio $d_{1} / d_{2}$ for which the Turing instability occurs, type of the resulting patterns, and possible biological implications. These questions have not been addressed before. Further, we would like to open a question if unilateral sources, which in mathematical models improve conditions for Turing instability and change the resulting form of patterns, may really exist in nature and whether they play a role in spatial patterning observed in biology.

Unilateral terms of the type $\hat{g}(v)=\tau v^{-}$have been introduced in the context of systems (1) under the assumptions guaranteeing the Turing instability already in [14. However, the stability of the ground state has not been analysed for this type of systems. This analysis is nontrivial, because the possible nonsmoothness of the unilateral term precludes the use of the standard linear analysis. Moreover, in Section 2 below we compare the system with nonsmooth unilateral term and systems, where the nonsmooth term is replaced by smooth approximations. Analytical results and numerical computations indicate that the ground state in the system with nonsmooth term is stable under different conditions than in systems with its smooth approximations. On the other hand, we also observe that perturbations larger than certain minimal size do evolve to qualitatively similar patterns under the same conditions for both the nonsmooth unilateral term and its smooth approximations. Thus, the fact whether a small perturbation of the ground state will evolve to a pattern or not is extremely sensitive to small changes of the nonlinear dynamics near the ground state. A small change of the term $\hat{g}$ in a neighbourhood of zero can turn the stability of the ground state to its instability and vice versa. However, the numerical case study presented in Subsection 2.3 indicates that if the initial perturbations of the ground state are larger than a certain minimal size then they robustly evolve to qualitatively similar patterns regardless small changes of the term $\hat{g}$ near zero.

Theoretically, it is not clear how to analyse the evolution of perturbations of the ground state that are larger than a certain minimal size. Such theory does not exist. However, the nonsmoothness of the unilateral term could help. We observe, at least in the particular examples presented in Section 2, that in cases when smooth approximations yield patterns for larger perturbations only, the nonsmooth term yields qualitatively similar patterns even from small perturbations. Thus, the question whether larger perturbations of the ground state will evolve to patterns in systems with (both smooth and nonsmooth) unilateral term or not seems to correspond to the question of stability with respect to small perturbations of the system with the nonsmooth unilateral term. Theoretical study of the question what are diffusion parameters for which spatially nonhomogeneous stationary solutions exist is done in [14] for the case of nonsmooth 
terms of the type $\tau v^{-}$, not for their smooth approximations. Further theoretical results about various other (nonsmooth) unilateral conditions can be found in above mentioned papers.

The rest of this paper is organized as follows. Section 2 shows the significance of the nonsmooth unilateral term $\hat{g}(v)=\tau v^{-}$and compares its influence on the initiation and final formation of spatial patterns with the influence of its smooth approximations. Section 3 presents numerical calculations showing spatial patterns produced by system (3) with $\hat{g}(v)=\tau v^{-}$, compares them with patterns obtained by the same system without any unilateral term, and shows how these patterns depend on the strength $\tau$ of the unilateral source and on the ratio of diffusion constants. We observe that unilateral terms yield asymmetric patterns with irregular spots. Concrete system (3) with the unilateral term $\hat{g}(v)=\tau v^{-}$ generates patterns even for greater ratio of diffusions in comparison with the classical system. Finally, we show that the difference between the patterns corresponding to the almost zero and high strength of the unilateral source resembles the difference between the roughly regular pattern of the common morph of the

cheetah and the irregular pattern of the king cheetah. Section 4 discusses the results and draws the conclusions.

\section{SignificAnCE of THE NONSMOOTH UnilaterAl TERM}

As we have already mentioned, the unilateral term need not be smooth at the point of the ground state and, therefore, the standard linear analysis cannot be performed, in general. If the unilateral term is non-smooth at the ground state, a natural idea is to approximate it by a smooth one. Such approximation can be arbitrarily precise and therefore we would expect that the behaviour of the approximate system will not considerably differ from the behaviour of that with the nonsmooth unilateral term. This vague statement is roughly correct from the perspective of the formation of the final pattern, but it is not true from the point of view of the Turing instability. The reason is that the stability is a local effect determined by small perturbations of the ground state, but the final pattern is formed by nonlinear terms $f, g$, and $\hat{g}$ evaluated at points $u$ and $v$ distant from the ground state. To illustrate this phenomenon, we provide a short case study to show how various approximations of the unilateral term may influence the Turing instability and what are their effects on the resulting patterns. Basically, we show that the occurrence of the Turing instability is extremely sensitive on small changes of the nonlinear dynamics near the ground state.

Particular system. We will discuss the particular system used in [4, 30] for the study of skin and coat patterns in fish and mammals, and supplement it by 
a unilateral source term $\hat{g}(v)$. Namely, we will consider the system

$$
\begin{aligned}
& \frac{\mathrm{d} u}{\mathrm{~d} t}=D \delta \Delta u+\alpha u+v-r_{2} u v-\alpha r_{3} u v^{2} \quad \text { in } \Omega, \\
& \frac{\mathrm{d} v}{\mathrm{~d} t}=\delta \Delta v-\alpha u+\beta v+r_{2} u v+\alpha r_{3} u v^{2}+\hat{g}(v) \quad \text { in } \Omega .
\end{aligned}
$$

Note that this system is a special case of (1) with $d_{1}=D \delta, d_{2}=\delta, f(u, v)=$ $\alpha u+v-r_{2} u v-\alpha r_{3} u v^{2}$, and $g(u, v)=-\alpha u+\beta v+r_{2} u v+\alpha r_{3} u v^{2}$. If $\hat{g}(v)=0$ then this system coincides with the original system from [4, 30] and we call it the classical case. As in [30], we will assume the homogeneous Neumann boundary conditions (2) and parameter values

$$
\delta=6, \alpha=0.899, \beta=-0.91, r_{2}=2, r_{3}=3.5 .
$$

For $D=0.45$ and $\hat{g}(v)=0$, these values yield the Turing diffusion driven instability [30], however, we will consider also different values of $D$.

Ground state. The ground state of system $(3)$ is defined in the same way as in Section 1, i.e. it consists of constants $\bar{u}, \bar{v}$ such that $f(\bar{u}, \bar{v})=g(\bar{u}, \bar{v})+\hat{g}(\bar{v})=0$. In particular, it can be readily verified that it is

$$
\bar{u}=-\bar{v} /\left(\alpha-r_{2} \bar{v}-\alpha r_{3} \bar{v}^{2}\right),
$$

where $\bar{v}$ is a root of the nonlinear equation

$$
(1+\beta) \bar{v}+\hat{g}(\bar{v})=0 .
$$

Clearly, if $\hat{g}(0)=0$ then $\bar{u}=\bar{v}=0$. This is the case for choices of $\hat{g}$ we are mainly interested in. However, certain choices of $\hat{g}$ introduced below do not vanish at zero and hence the corresponding ground state is nonzero.

Conditions for the Turing instability. In the case when the additional unilateral term $\hat{g}(v)$ in $(3)$ is smooth at $\bar{v}$, we can perform the standard linear analysis to obtain necessary conditions for the Turing instability, see e.g. [12, 20, 32. Namely, we can introduce the Jacobi matrix of the map $f, g+\hat{g}$ at $\bar{u}, \bar{v}$ as

$$
B=\left[\begin{array}{ll}
b_{11}, & b_{12} \\
b_{21}, & b_{22}
\end{array}\right]=\left[\begin{array}{cc}
\partial f / \partial u, & \partial f / \partial v \\
\partial g / \partial u, & \partial g / \partial v+\mathrm{d} \hat{g} / \mathrm{d} v
\end{array}\right](\bar{u}, \bar{v})
$$

If

$$
\operatorname{tr} B<0 \text { and } \operatorname{det} B>0,
$$

then the ground state $(\bar{u}, \bar{v})$ is asymptotically stable with respect to small spatially homogeneous perturbations. If simultaneously

$$
b_{11} b_{22}<0 \text { and } b_{12} b_{21}<0
$$

then this ground state is stable (with respect to small spatially nonhomogeneous perturbations) only for some values of $D$ and unstable for the others, see e.g. [32, sec. 2.3].

Critical ratio of diffusions. Parameter values (4) are chosen in such a way that for $\hat{g} \equiv 0$ conditions (8) and (9) are fulfilled. In any case, if $\hat{g}(v)$ is smooth 
at $\bar{v}$ and if conditions (8) and $(9)$ hold then a necessary condition for the ground state of system (3) to be unstable with respect to spatially nonhomogeneous perturbations is that the ratio of diffusion coefficients $D$ is sufficiently small. Precisely, the condition is

$$
D<D_{\text {crit }} \quad \text { with } \quad D_{\text {crit }}=\frac{1}{b_{22}^{2}}\left(\operatorname{det} B-b_{12} b_{21}-2 \sqrt{-b_{12} b_{21} \operatorname{det} B}\right) .
$$

Note that the definition of $D_{\text {crit }}$ in $(10)$ is just a reciprocal value of the formula from [33, p. 562]. It can also be easily derived from the analysis of [32, p. 109]. In any case, if condition (10) is not satisfied then the Turing instability cannot occur. It is essential that if $\hat{g}$ is not smooth at the ground state value $\bar{v}$ then this linear analysis cannot be performed. Jacobian $B$ is simply not defined and consequently formula 10 has no sense. The critical ratio $D_{\text {crit }}$ can be only estimated numerically.

The forthcoming Subsection 2.1 discusses the stability of the ground state with respect to spatially homogeneous perturbations, i.e. the stability for the system without diffusion. Subsection 2.2 defines six particular choices of $\hat{g}(v)$ and compares them with respect to the Turing instability. We emphasize that the Turing instability is a local phenomenon determined by small perturbations and hence only the values of $\hat{g}(v)$ in a small neighbourhood of the ground state $\bar{v}$ are relevant. We will see that although the six choices of $\hat{g}(v)$ differ only slightly in the neighbourhood of the ground state, some of them yield patterns evolving from small perturbations and some of them do not. However, further in Subsection 2.3 we will see that if the perturbations of the ground state are larger than a certain minimal size, then they evolve to qualitatively similar patterns in all cases.

We note that all patterns in this paper are computed numerically by our own Matlab based finite element solver. The convergence and stability of the finite element method is well known [9]. Its convergence for the specific problem (3) is analysed in [28]. The time stepping is done by the build in Matlab ODE solver, which stops as soon as the prescribed final time is reached. We set this time to $5 \cdot 10^{4}$ for all presented calculations. This is a sufficiently high value, because the experimentally determined times of reaching the steady state are usually around $2-3 \cdot 10^{3}$, at most $10^{4}$. The high value of the final time does not increase the total computational time considerably, because the method determines the time step adaptively. As soon as the numerical method detects the steady state, the time step quickly increases and the final time is reached in a few iterations. The initial condition is always chosen as small random fluctuations around the ground state, except of Figure 3, where the fluctuations are larger. Clearly, different initial conditions may and often do evolve to different stationary solutions, but qualitative features of these solutions are the same. We choose the domain to be $\Omega=(-100,100)^{2}$ and in the subsequent figures, we plot the patterns as graphs of the solution component $u$, where values of $u$ are indicated by shades of grey. We 
do not plot the component $v$, because it is complementary to $u$, cf. [32, p. 88], and patterns based on $v$ are almost exact inverses of patterns based on $u$.

2.1. Stability for systems without diffusion. In this subsection, we consider system (3) without the diffusion terms and we analyse the stability of its ground state. We are mainly interested in the case $\hat{g}(v)=\tau v^{-}$, i.e. in the natural choice $\theta=0$, and study the system

$$
\begin{aligned}
& \frac{\mathrm{d} u}{\mathrm{~d} t}=\alpha u+v-r_{2} u v-\alpha r_{3} u v^{2}, \\
& \frac{\mathrm{d} v}{\mathrm{~d} t}=-\alpha u+\beta v+r_{2} u v+\alpha r_{3} u v^{2}+\tau v^{-} .
\end{aligned}
$$

The ground state of this system is $(\bar{u}, \bar{v})=(0,0)$ and its stability cannot be investigated by the standard linearisation due to the nonsmoothness of the unilateral term. Nevertheless, we can prove it using, for example, the theory of Filippov systems [17].

Lemma 1. If $\alpha, \beta$ are given by (4) and $\tau \in(0,0.09)$ then the trivial solution $(\bar{u}, \bar{v})=(0,0)$ of system (11) is asymptotically stable.

Proof. Let us start with the system without higher order terms, i.e.

$$
\frac{\mathrm{d} u}{\mathrm{~d} t}=\alpha u+v, \quad \frac{\mathrm{d} v}{\mathrm{~d} t}=-\alpha u+\beta v+\tau v^{-} .
$$

Let $U(t)=(u(t), v(t))$ be its arbitrary solution. If $\alpha, \beta$ are given by (4) and $t_{0}$ is such that $v\left(t_{0}\right)=0$ and $u\left(t_{0}\right)>0$, then it follows from $\sqrt{12}$ that $\frac{\mathrm{d} u}{\mathrm{~d} t}\left(t_{0}\right)>0$ and $\frac{\mathrm{d} v}{\mathrm{~d} t}\left(t_{0}\right)<0$. Similarly, if $v\left(t_{0}\right)=0$ and $u\left(t_{0}\right)<0$ then $\frac{\mathrm{d} u}{\mathrm{~d} t}\left(t_{0}\right)<0$ and $\frac{\mathrm{d} v}{\mathrm{~d} t}\left(t_{0}\right)>0$. Thus, the solution intersects the axis $v=0$ transversally and the whole time interval $(0,+\infty)$ consists of open intervals where $v>0$, open intervals where $v<0$, and isolated points. In time intervals where $v(t)>0$, the solution $U(t)$ coincides with a solution of the linear system obtained by the choice $\tau=0$, and in time intervals where $v(t)<0$, it coincides with a solution of the linear system

$$
\frac{\mathrm{d} u}{\mathrm{~d} t}=\alpha u+v, \quad \frac{\mathrm{d} v}{\mathrm{~d} t}=-\alpha u+\beta v-\tau v .
$$

For $\alpha, \beta$ given by (4), the matrix $B_{0}$ of system (12) with $\tau=0$ satisfies (8). The trace of the matrix $B_{\tau}$ of system (13) is negative for all $\tau>-0.011$ and its determinant is positive for $\tau<0.09$. It follows that for $\tau \in(0,0.09)$, the eigenvalues of both matrices $B_{0}$ and $B_{\tau}$ have negative real parts, that means the trivial solution of both linear systems is asymptotically stable. Due to the form of solutions of linear systems with constant coefficients, there exists $C>0$ such that if $U(t)$ is any solution of $(12)$ with $\tau=0$ or $(13)$ then

$$
\|U(t)\| \leq C\|U(0)\| \exp (\Lambda t)
$$

where $\Lambda$ is the maximum over real parts of all eigenvalues of both matrices $B_{0}$ and $B_{\tau}$. Using considerations above we see that also the trivial solution of (12) is 
asymptotically stable. Further, it follows from known results concerning systems with nonsmooth right hand sides that then the trivial solution of the full system (11) with higher order terms is also asymptotically stable, see e.g. [17, p. 169, Theorem 7].

We note that in fact the trivial solution of (11) is stable for a larger interval of $\tau$ because its stability for both linear problems mentioned is only sufficient, not necessary condition. Further we note that in all situations discussed below we will have $\tau<0.09$ and therefore the trivial solution of our problem without any diffusion will be always stable.

As we already mentioned, we will also investigate the effect of smoothing of the nonsmooth unilateral term $\tau v^{-}$. We will consider several approximations in the form $\hat{g}(v)=\tau n_{\varepsilon}(v)$, where the parameter $\varepsilon>0$ controls the accuracy of the approximation. Functions $n_{\varepsilon}(v)$ are smooth in a neighbourhood of the ground state and approximate $v^{-}$in the sense that $n_{\varepsilon}(v)$ converge to $v^{-}$as $\varepsilon \rightarrow 0_{+}$. This setting enables to analyse the stability of the ground state for all approximations of this type at once.

Thus, we consider the ODE system

$$
\begin{aligned}
& \frac{\mathrm{d} u}{\mathrm{~d} t}=\alpha u+v-r_{2} u v-\alpha r_{3} u v^{2}, \\
& \frac{\mathrm{d} v}{\mathrm{~d} t}=-\alpha u+\beta v+r_{2} u v+\alpha r_{3} u v^{2}+\tau n_{\varepsilon}(v),
\end{aligned}
$$

where the parameter values are given in (4) and $\tau \geq 0, \varepsilon>0$ are free parameters. We assume that (6) with $\hat{g}(v)=\tau n_{\varepsilon}(v)$ has a unique solution $\bar{v}_{\varepsilon}$ for any $\varepsilon>0$. This $\bar{v}_{\varepsilon}$ together with $\bar{u}_{\varepsilon}$ given by (5) forms the ground state of (14).

Lemma 2. Let $\left(\bar{u}_{\varepsilon}, \bar{v}_{\varepsilon}\right)$ be the ground state of system (14). For any $\varepsilon>0$, let the function $n_{\varepsilon}$ be smooth in a neighbourhood of $\bar{v}_{\varepsilon}$ and let $n_{\varepsilon}^{\prime}(v)$ denote the derivative of $n_{\varepsilon}(v)$. If $n_{\varepsilon}(v)$ converges uniformly in $\mathbb{R}$ to $v^{-}$as $\varepsilon \rightarrow 0_{+}$and if

$$
\tilde{n}:=\lim _{\varepsilon \rightarrow 0_{+}} n_{\varepsilon}^{\prime}\left(\bar{v}_{\varepsilon}\right) \text { exists, } \quad \tilde{n} \leq 0, \quad \text { and } \quad 0 \leq \tau<(1+\beta) \min \{1,-1 / \tilde{n}\},
$$

then the ground state $\left(\bar{u}_{\varepsilon}, \bar{v}_{\varepsilon}\right)$ of system (14) is asymptotically stable for all sufficiently small $\varepsilon>0$.

Proof. First, we notice that the ground state tends to zero as $\varepsilon \rightarrow 0_{+}$. This clearly follows from (5) and the convergence $\bar{v}_{\varepsilon} \rightarrow 0$ which can be proved by contradiction. Indeed, if $\bar{v}_{\varepsilon}$ did not converge to 0 then there would be a sequence $\varepsilon_{k} \rightarrow 0$ such that $\bar{v}_{\varepsilon_{k}} \rightarrow v_{0}$ with $v_{0} \neq 0$. Due to (6), we would have the identity

$$
0=(1+\beta) \bar{v}_{\varepsilon_{k}}+\tau n_{\varepsilon_{k}}\left(\bar{v}_{\varepsilon_{k}}\right)
$$

If $v_{0}$ were finite then using the uniform convergence $n_{\varepsilon}(v) \rightarrow v^{-}$we would get $(1+\beta) v_{0}+\tau v_{0}^{-}=0$. However, this equation has only the zero solution because $\tau<1+\beta$. This would contradict the assumption $v_{0} \neq 0$. It is not hard to see, 
again by using the uniform convergence and the assumption $\tau<1+\beta$, that if $v_{0}$ were not finite then (16) could not hold for large $k$.

Second, we show the asymptotic stability. We linearise system (14) around $\left(\bar{u}_{\varepsilon}, \bar{v}_{\varepsilon}\right)$. The matrix $B_{\varepsilon}$ of this linearisation is given by $(7)$ with $f(u, v)=\alpha u+$ $v-r_{2} u v-\alpha r_{3} u v^{2}, g(u, v)=-\alpha u+\beta v+r_{2} u v+\alpha r_{3} u v^{2}+\hat{g}(v), \hat{g}(v)=\tau n_{\varepsilon}(v)$, and $(\bar{u}, \bar{v})=\left(\bar{u}_{\varepsilon}, \bar{v}_{\varepsilon}\right)$. Due to convergences $\left(\bar{u}_{\varepsilon}, \bar{v}_{\varepsilon}\right) \rightarrow(0,0)$ and $n_{\varepsilon}^{\prime}\left(\bar{v}_{\varepsilon}\right) \rightarrow \tilde{n}$, we have

$$
\lim _{\varepsilon \rightarrow 0_{+}} B_{\varepsilon}=\left[\begin{array}{cc}
\alpha, & 1 \\
-\alpha, & \beta+\tau \tilde{n}
\end{array}\right] .
$$

Under the assumption $0 \leq \tau<-(1+\beta) / \tilde{n}$, we can easily verify that this matrix satisfies conditions (8). Thus, the continuity argument implies that even the matrix $B_{\varepsilon}$ satisfies conditions (8) for sufficiently small $\varepsilon>0$ and, consequently, the stationary solution $\left(\bar{u}_{\varepsilon}, \bar{v}_{\varepsilon}\right)$ is asymptotically stable.

2.2. Turing instability for various choices of $\hat{g}(v)$. Now, we will consider several particular choices of $\hat{g}(v)$ and compare their influence on the Turing instability, i.e. on the evolution of small spatially nonhomogeneous perturbations of the ground state. The idea is to consider the unilateral source term $\hat{g}(v)=\tau v^{-}$ as the reference choice and the other cases are seen as its approximations. As a criterion for the comparison we choose the critical ratio $D_{\text {crit }}$, see $(10)$ and the discussion below (10). We will see that $D_{\text {crit }}$ varies considerably for different choices of $\hat{g}(v)$ and that this variation is essential even in the case of very accurate approximations. Note that the strength of the unilateral source is

$$
\tau=0.075
$$

for all cases throughout this section. Now, we list the choices of $\hat{g}(v)$ we make.

(a) Nonsmooth unilateral source, $\hat{g}(v)=\tau v^{-}$, see Figure 1(a).: This is the reference case. The ground state of system (3) with this choice of $\hat{g}(v)$ is $(\bar{u}, \bar{v})=(0,0)$. Its asymptotic stability with respect to small spatially homogeneous perturbations, i.e. as a stationary solution of ODE system (11), follows from Lemma 1. The instability of this ground state as a solution of (3) cannot be investigated by the linear analysis, but numerical calculations indicate that spatially nonhomogeneous perturbations as small as we can afford numerically, evolve to nonhomogeneous stationary solutions for the ratio of diffusions below $D_{\text {crit }}^{(\mathrm{a})}=0.71$. Note that this value is greater than the critical ratio of diffusion for the classical case $(\hat{g} \equiv 0)$, which is $D_{\text {crit }}=0.53$. See Section 3 for more details.

(b) Smooth quadratic approximation.: The nonsmooth function from the previous case can be smoothed for example as

$$
\hat{g}(v)=\left\{\begin{array}{cc}
\tau(v-\varepsilon)^{2} /(4 \varepsilon) & \text { for }|v|<\varepsilon \\
\tau v^{-} & \text {for }|v| \geq \varepsilon
\end{array}\right.
$$


(a)

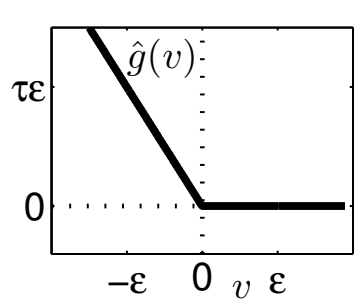

(d)

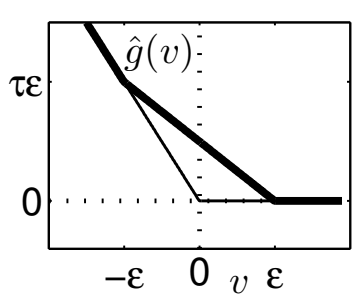

(b)

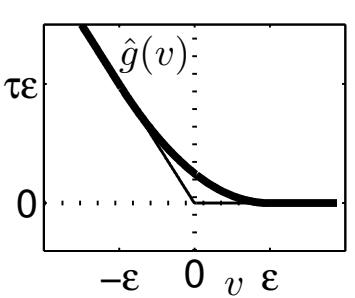

(e)

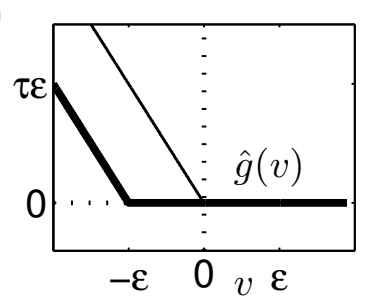

(c)

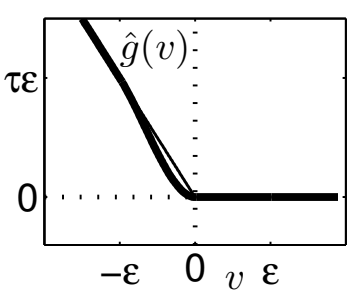

(f)

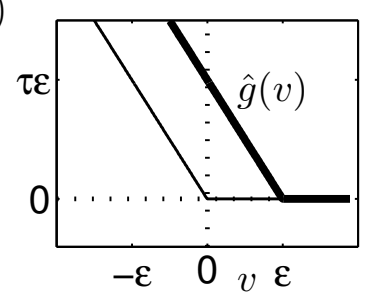

FiguRE 1. Graphs of different choices of the unilateral term $\hat{g}(v)$ in $(3)$ : (a) $\hat{g}(v)=\tau v^{-}$, (b) $\hat{g}(v)$ given by (18), (c) $\hat{g}(v)$ given by (19), (d) $\hat{g}(v)$ given by $(20)$, (e) $\hat{g}(v)=\tau(v+\varepsilon)^{-}$, (f) $\hat{g}(v)=\tau(v-\varepsilon)^{-}$. Thin lines show the graph of $\tau v^{-}$for comparison.

where $\varepsilon>0$ is a small parameter, see Figure 1(b). System (3) with (2) and this choice of $\hat{g}(v)$ has the ground state with $\bar{u}_{\varepsilon}$ given by (5) and

$$
\bar{v}_{\varepsilon}=2 \frac{\varepsilon}{\tau}\left(-1-\beta+\frac{\tau}{2}+\sqrt{(1+\beta)(1+\beta-\tau)}\right) .
$$

For the chosen parameters, assumptions (15) in Lemma 2 are satisfied, because, in particular, $\tilde{n} \approx-0.71$. Thus, the asymptotic stability of $\left(\bar{u}_{\varepsilon}, \bar{v}_{\varepsilon}\right)$ of (14) follows for sufficiently small $\varepsilon>0$. Moreover, numerically we can easily find that it is asymptotically stable for $\varepsilon<0.038$. The corresponding critical ratio of diffusions can be expressed from (10) for any $0<\varepsilon<0.038$ and we plot its values in Figure 2. In the limit $\varepsilon \rightarrow 0_{+}$ we have $D_{\text {crit }}^{(\mathrm{b})} \approx 0.63$.

(c) Smooth cubic approximation.: Another option how to smooth the function from the case (a) is

$$
\hat{g}(v)=\left\{\begin{array}{cl}
\tau v^{2}(v+2 \varepsilon) / \varepsilon^{2} & \text { for }-\varepsilon<v<0, \\
\tau v^{-} & \text {for } v \leq-\varepsilon \text { and } v \geq 0,
\end{array}\right.
$$

where $\varepsilon>0$ is again a small parameter, see Figure 1(c). The ground state in this case is $(\bar{u}, \bar{v})=(0,0)$ independently of $\varepsilon$ and since the derivative of $\hat{g}$ at zero vanishes, the critical ratio of diffusions is the same as in the classical system $(\hat{g} \equiv 0)$. Using 10 we obtain $D_{\text {crit }}^{(\mathrm{c})}=D_{\text {crit }} \approx 0.53$.

(d) Linear cut.: The choice (a) can be approximated by a continuous piecewise linear function such that it is smooth at the ground state. A 
straightforward choice is

$$
\hat{g}(v)=\left\{\begin{array}{cc}
\tau(\varepsilon-v) / 2 & \text { for }|v|<\varepsilon, \\
\tau v^{-} & \text {for }|v| \geq \varepsilon,
\end{array}\right.
$$

see Figure 1(d). The ground state is shifted away from zero. Its component $\bar{u}_{\varepsilon}$ is given by (5) and

$$
\bar{v}_{\varepsilon}=\frac{\tau \varepsilon}{\tau-2-2 \beta} .
$$

Using Lemma 2 for parameter values (4) and (17), we obtain the asymptotic stability of the ground state $\left(\bar{u}_{\varepsilon}, \bar{v}_{\varepsilon}\right)$ of (14) for sufficiently small $\varepsilon>0$, because $\tilde{n}=-1 / 2$ and assumptions (15) are satisfied. Numerically, we verify that it is asymptotically stable for $\varepsilon \leq 0.016$. The critical ratio of diffusions is computed from 10 and its dependence on $\varepsilon$ is illustrated in Figure 2. The limit value for $\varepsilon \rightarrow 0_{+}$is $D_{\text {crit }}^{(\mathrm{d})} \approx 0.60$.

(e) Shift of the threshold to the left, $\hat{g}(v)=\tau(v+\varepsilon)^{-}$, see Figure $1(\mathbf{e})$.: The corresponding ground state is $(\bar{u}, \bar{v})=(0,0)$ for all $\varepsilon>0$. This $\hat{g}(v)$ is smooth at zero and both its value and derivative at zero vanish. Therefore, conditions for the Turing instability are the same as in the classical case $\hat{g} \equiv 0$ and formula $(10)$ provides the same critical ratio of diffusions as in the case $(\mathrm{c})$, i.e. $D_{\text {crit }}^{(\mathrm{e})}=D_{\text {crit }}^{(\mathrm{c})}=D_{\text {crit }} \approx 0.53$.

(f) Shift of the threshold to the right, $\hat{g}(v)=\tau(v-\varepsilon)^{-}$, see Figure 1(f).: This choice yields a nonzero ground state $\bar{u}_{\varepsilon}$ given by (5) and

$$
\bar{v}_{\varepsilon}=\frac{\tau \varepsilon}{\tau-1-\beta} .
$$

Using Lemma 2 for parameters (4) and (17), we obtain the asymptotic stability of the ground state $\left(\bar{u}_{\varepsilon}, \bar{v}_{\varepsilon}\right)$ of $(14)$ for sufficiently small $\varepsilon>0$, because $\tilde{n}=-1$ and assumptions (15) are satisfied. Numerically, we verify that it is asymptotically stable for $\varepsilon<0.0044$. The critical ratio of diffusions can be obtained from 110 . Its values are provided in Figure 2 and its limit for $\varepsilon \rightarrow 0_{+}$is $D_{\text {crit }}^{(\mathrm{f})} \approx 0.71$.

To compare choices (a)-(f), we summarize the dependence of the critical ratio of diffusions on $\varepsilon$ in Figure 2. Note that the accuracy of approximations (b)-(f) of the reference choice (a) is controlled by $\varepsilon$. Smaller $\varepsilon$ corresponds to more accurate approximations. We clearly observe that different choices of $\hat{g}(v)$ yield considerably different critical ratios of diffusions and, hence, various approximations of the nonsmooth unilateral term $\tau v^{-}$exhibit the Turing instability for different values of the ratio $D$. For example, if $\varepsilon=0.005$ and $D=0.65$ (see the grey diamond in Figure 2), then choice (a) is the only case which exhibits the Turing instability. Indeed, in cases (b)-(e) the ground state is stable with respect to all small perturbations, because the ratio of diffusion $D=0.65$ is above the critical value. In the case (f) the Turing instability cannot occur, because $\operatorname{tr} B$ is positive 


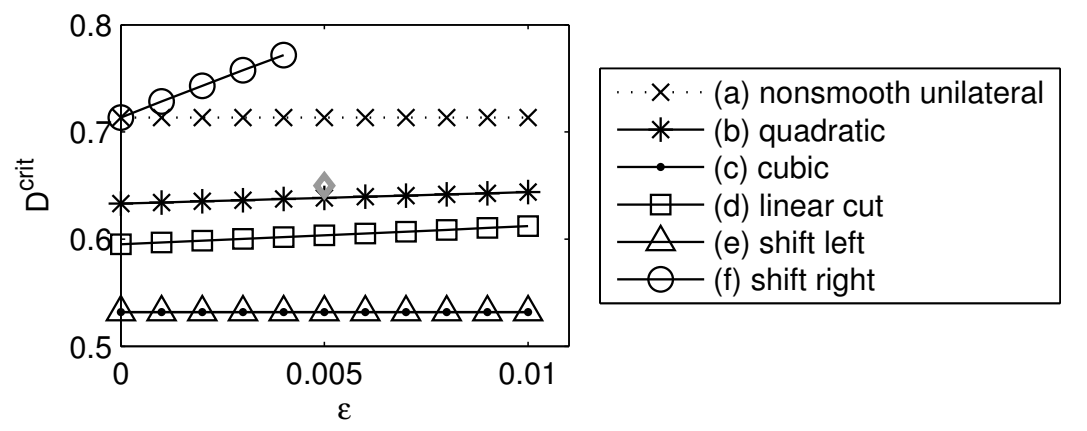

Figure 2. Dependence of the critical ratio of diffusions $D_{\text {crit }}$ on $\varepsilon$ for choices (a)-(f). The value for choice (a) is estimated numerically and the other values are computed by (10). Notice that cases (c) and (e) coincide. In case (f), the critical ratio is not defined for $\varepsilon$ above approximately 0.0044 , because then the trace of $B$ is positive, see (8). The grey diamond indicates the case of parameter values $\varepsilon=0.005$ and $D=0.65$ for which Turing patterns appear in the case (a) only.

for $\varepsilon>0.0044$ and therefore the ground state is unstable even with respect to spatially homogeneous perturbations. Similarly, if we decrease $\varepsilon$ to 0.001 and keep $D=0.65$, then the choices (a) and (f) exhibit the Turing instability, but choices (b)-(e) do not.

Note that our statement that the choice (a) exhibits the Turing instability is based on numerical calculations, where we observe that small perturbations of the ground state evolve to patterns. For more details and numerical results, see Figure 5 below, the panels for $\tau=0.075$.

Figure 2 clearly shows the size of variations in $D_{\text {crit }}$ for different approximations of the nonsmooth unilateral source term. Even if we arbitrarily increase the accuracy of these approximations, i.e. in the limit $\varepsilon \rightarrow 0$, the corresponding values of $D_{\text {crit }}$ differ considerably. Hence, each approximation yields the Turing instability for different ranges of diffusion coefficients. Consequently, the idea to approximate the nonsmooth term by a smooth one and analyse it by standard means fails. Simple smooth approximations of the nonsmooth unilateral term with accuracy controlled by $\varepsilon$ can yield misleading results in the limit $\varepsilon \rightarrow 0$.

From the biological perspective, all choices (a)-(f) seem to be plausible. Although some of these choices have the threshold value shifted from the ground state, the difference is not large.

Of course, we could also discuss other approximations of the nonsmooth unilateral term. However, results of the next subsection indicate that these approximations result to the same (or very similar) patterns as the unilateral term $\hat{g}(v)=\tau v^{-}$, provided the other parameters of the problem are the same. At the same time, it is important to mention that not all of these approximations yield 
(a)

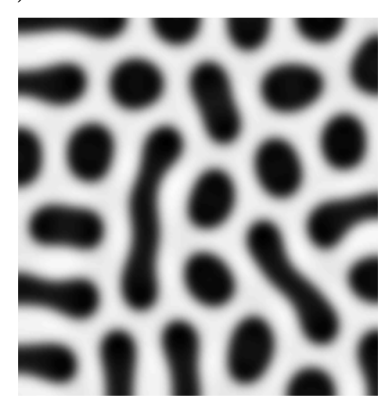

(d)

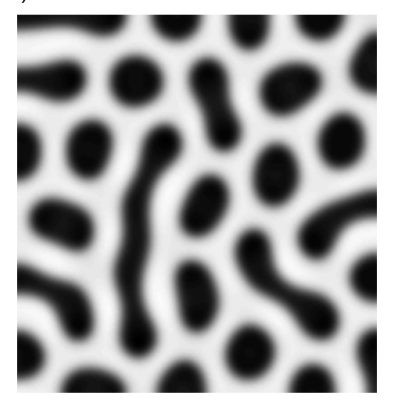

(b)

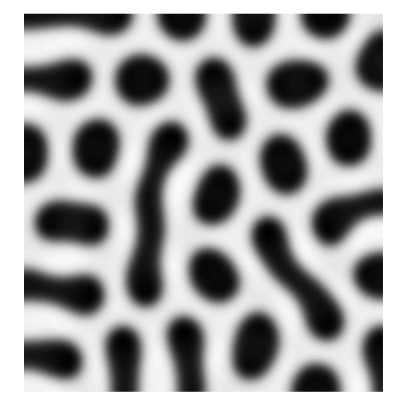

(e)

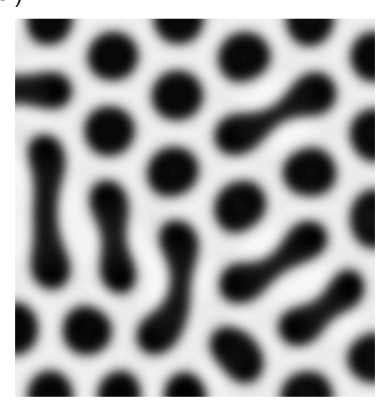

(c)

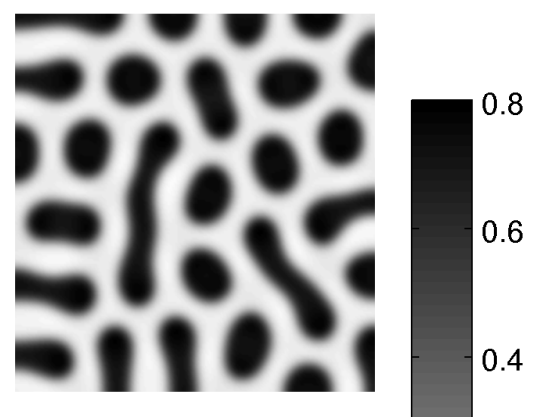

(f)

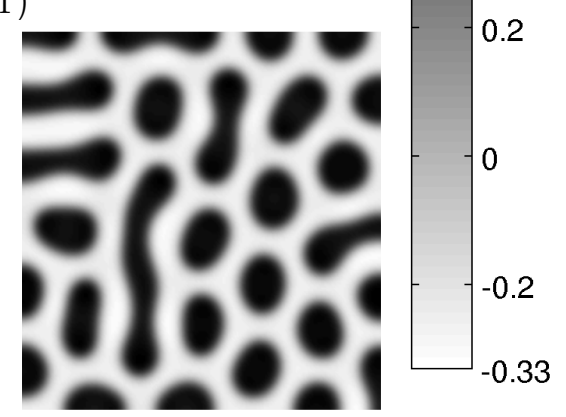

FiguRE 3. Patterns produced from larger perturbations of the ground state in cases (a)-(f) with $D=0.65, \varepsilon=0.005, \tau=0.075$, and parameter values (4). The initial condition is the same in all cases and consists of random disturbances of the ground state with maximal amplitude $20 \varepsilon=0.1$. Note that initial conditions with maximal amplitude of size $\varepsilon$ or smaller evolve to patterns in case (a) only.

patterns developing from small perturbations. Sometimes, the perturbations have to be sufficiently distant from the ground state, as we describe below.

2.3. Patterns for various choices of $\hat{g}(v)$. Above, we introduced an example of parameter values for system (3) such that small perturbations of the ground state do not evolve to any patterns in cases (b)-(f), but they do in the case (a), see the grey diamond in Figure 2. Now, we will see that perturbations larger than a certain minimal size (depending on $\varepsilon$ ) do evolve to patterns in all these cases. We also show that all these patterns are qualitatively the same. Moreover, if they evolve from the same initial condition, they are all also quantitatively very similar and some of them are even exactly identical, see Figure 3 .

Similarity of these patterns is not surprising, because the differences among all choices of $\hat{g}(v)$ in cases (a)-(f) are insignificant on scales considerably larger than $\varepsilon$. Since the magnitude of the final pattern (i.e. the stationary solution to (3)) 
is of order one and the size of $\varepsilon$ is of order one thousands, we can expect similar patterns in all these cases.

As we have mentioned, small perturbations of the ground state do not evolve to any patterns in cases (b)-(f) for the chosen values $D=0.65$ and $\varepsilon=0.005$. This fact follows from the linear analysis and we observe it numerically as well. However, Figure 3 shows that perturbations that are larger than a certain minimal size, do evolve to patterns in all these cases. Moreover, we observe identical patterns for choices (a)-(c) and a very similar pattern for the choice (d). Choices (e) and (f) yield slightly more distinct patterns, but they share the same qualitative features as the other cases.

We also computed the patterns starting from an initial condition twice as large as was used in Figure 3 and we obtained identical patterns for all cases (a)(d). (These results are not presented.) Patterns (e) and (f) were different in a similar manner as in Figure 3. This is understandable, because choices (a)-(d) of $\hat{g}(v)$ are identical for $|v| \geq \varepsilon$ and thus if the size of the initial condition is sufficiently large, the influence of $\hat{g}(v)$ for $|v| \geq \varepsilon$ overweights the influence of $\hat{g}(v)$ for $|v|<\varepsilon$ and identical patterns emerge. On the contrary, in cases (e) and (f) the values $\hat{g}(v)$ slightly differ even for $|v| \geq \varepsilon$ and therefore the resulting patterns differ as well. Hence, for the particular system (3) it seems that if two nonlinear kinetics differ on a small neighbourhood of the (unique) ground state only then there exists (almost) the same pattern for both kinetics and sufficiently large initial perturbations of the ground state will evolve to this pattern for both kinetics. If this statement is true then practically relevant is the evolution of perturbations greater than a certain minimal size rather than the evolution of small perturbations. The reason is the robustness of the evolution of the larger perturbations to patterns observed in numerical tests described in this section and the fact that the stability with respect to the small perturbations is highly sensitive to small changes of $\hat{g}(v)$ in the neighbourhood of the ground state.

Importantly, the nonsmooth unilateral term (a) yields patterns that evolve from small spatial perturbations for a large range of values of $D$, as far as we can conclude from numerous numerical calculations we performed. This is the essential motivation to investigate the nonsmooth unilateral case (a). It provides predictions about a whole class of approximations of the nonsmooth term $\hat{g}(v)=\tau v^{-}$. The tested choices (b)-(f) are just examples of members of this class. All approximations from this class produce the desired patterns and all these patterns are similar, however, for certain approximations the patterns do not evolve from small perturbations. There is no known theory so far that would explain the evolution of initial perturbations that are not small. However, the approaches presented in [14, 23, 27] provide certain ideas how to treat theoretically the positive homogeneous nonsmooth case $\hat{g}(v)=\tau v^{-}$. And, as we have already mentioned, the stability and instability of the ground state in systems 
with this term seem to correspond to the question whether the larger perturbations of the ground state do evolve to patterns or not for systems where this term is approximated.

\section{EXISTENCE OF PATTERNS AND THEIR DEPENDENCE ON PARAMETERS}

In this section we further investigate system (3) with the nonsmooth unilateral term $\hat{g}(v)=\tau v^{-}$to show when the Turing instability occurs, what is the effect of this term on the resulting patterns, and how they depend on the strength $\tau$ and on the ratio of diffusions $D$. In addition, we numerically compare behaviour of the system with this nonsmooth unilateral term with cases $\hat{g} \equiv 0$ and $\hat{g}(v)=-\tau v$. Comparing to these linear choices of $\hat{g}(v)$, we show that the unilateral term produces irregular patterns. Further, we present numerical results indicating that system (3) with the nonsmooth term $\hat{g}(v)=\tau v^{-}$yields patterns for considerably higher ratio of diffusion constants comparing to the classical system with $\hat{g} \equiv 0$. In addition, the choice $\hat{g}(v)=-\tau v$ seems to be informative about the Turing instability of the nonsmooth unilateral term. For system (3), we present results of numerical calculations supporting the hypothesis that the Turing instability occurs in the nonsmooth unilateral case $\hat{g}(v)=\tau v^{-}$for the same ratio of diffusion coefficients as for the choice $\hat{g}(v)=-\tau v$. Finally, in the last part of this section, we compare the patterns obtained with the nonsmooth unilateral term with the coat pattern of king cheetah and suggest a mechanism generating this pattern.

3.1. Unilateral term yields irregular patterns. First, we compare the patterns produced by the nonsmooth unilateral term and by the linear terms $\hat{g} \equiv 0$ and $\hat{g}(v)=-\tau v$. To this end we consider system (3) with boundary conditions (2), and parameter values (4). Figure 4 compares patterns for choices $\hat{g}(v)=\tau v^{-}$, $\hat{g} \equiv 0$, and $\hat{g}(v)=-\tau v$, respectively, for $\tau=0.08$ and $D=0.45$. Comparing these patterns we immediately observe the qualitative difference. The linear choices of $\hat{g}(v)$ produce approximately circular spots which are, to some extent, symmetrically placed. In contrast, the pattern produced by the unilateral system shows irregular spots of larger size. Several of the largest spots seem to be created by fusions of smaller spots. Moreover, the pattern does not exhibit any symmetry even approximately. Interestingly, similar irregular patterns are obtained in 39] by varying the parameter $h$ in the dimensionless version of model (3) without any unilateral source.

3.2. Critical ratio of diffusions. Another interesting phenomenon resulting from the addition of the nonlinear unilateral source terms to the classical system (i.e. (3) with $\hat{g} \equiv 0$ ) is the growth of small nonhomogeneous perturbations of the ground state to patterns even if the ratio of diffusions exceeds the critical value $(10)$ of the classical system (i.e. $\hat{g} \equiv 0$ ). Indeed, the critical ratio of diffusions (10) for the classical system with parameter values (4) is $D_{\text {crit }} \approx 0.53$. However, using the nonsmooth unilateral source $\hat{g}(v)=\tau v^{-}$, we numerically 

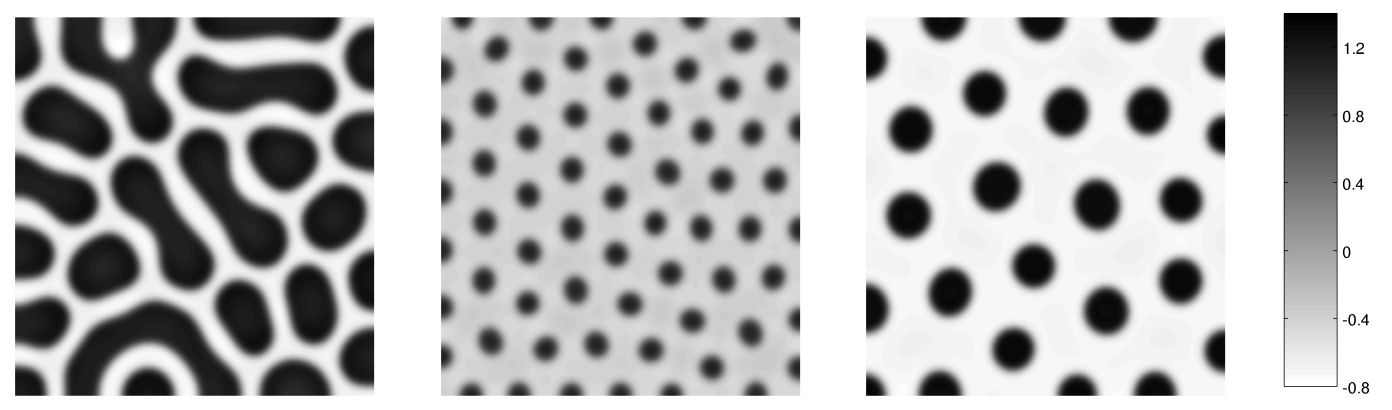

Figure 4. Typical patterns obtained by system (3) with the nonsmooth unilateral term $\hat{g}(v)=\tau v^{-}$(left panel), the classical case $\hat{g} \equiv 0$ (middle panel), and $\hat{g}(v)=-\tau v$ (right panel) with $\tau=0.08, D=0.45$, and parameter values (4). The initial condition was specified as a small random noise around the ground state. The grey scale shows the values of $u$.

obtain patterns forming from very small spatial perturbations of the ground state even for considerably higher ratios of diffusions. This phenomenon was predicted by a series of theoretical results, mainly [23, 27].

In order to illustrate the dependence of the arising patterns on the strength of the unilateral source $\tau$ and on the ratio of diffusion constants $D$, we present Figure 5. The top-left box in Figure 5 corresponds to the classical system $(\hat{g} \equiv 0)$ with standard parameter values (4) and $D=0.45$. We observe the typical regular spotted pattern. As $\tau$ increases, the spots are growing bigger and starting from certain value they seem to merge and irregular patterns emerge. Similarly, we can observe that higher values of $\tau$ enable to produce patterns for higher ratios of diffusions $D$. In particular, columns $3-5$ show that if $D$ exceeds the critical ratio of diffusions $D_{\text {crit }} \approx 0.53$ of the classical system, then the spatial patterns arise only if $\tau$ is sufficiently large. The larger is $D$, the larger $\tau$ is necessary for patterns to arise. For completeness, we mention that numerically no patterns emerge for $\tau \geq 0.089$.

3.3. Linear term $\hat{g}=-\tau v$. It is interesting to compare the above results with the case $\hat{g}(v)=-\tau v$. Note that this choice can actually be seen as the classical system with $\hat{g} \equiv 0$ and coefficient $\beta$ modified to $\beta-\tau$. Figure 6 shows the resulting patterns for various values of $D$ and $\tau$. This system is smooth and therefore we can analyse the Turing instability including the critical ratios of diffusion coefficients (10). Table 1 presents these values for parameters (4) and various $\tau$. Figure 6 confirms that this system produces patterns only if the ratio of diffusion coefficients is below the critical value. Interestingly, we observe patterns for the same values of the ratio of diffusions as for the system with $\hat{g}(v)=\tau v^{-}$ presented in Figure 5. This leads us to a hypothesis that the Turing instability in the unilateral system (3) with $\hat{g}(v)=\tau v^{-}$occurs under the same conditions 


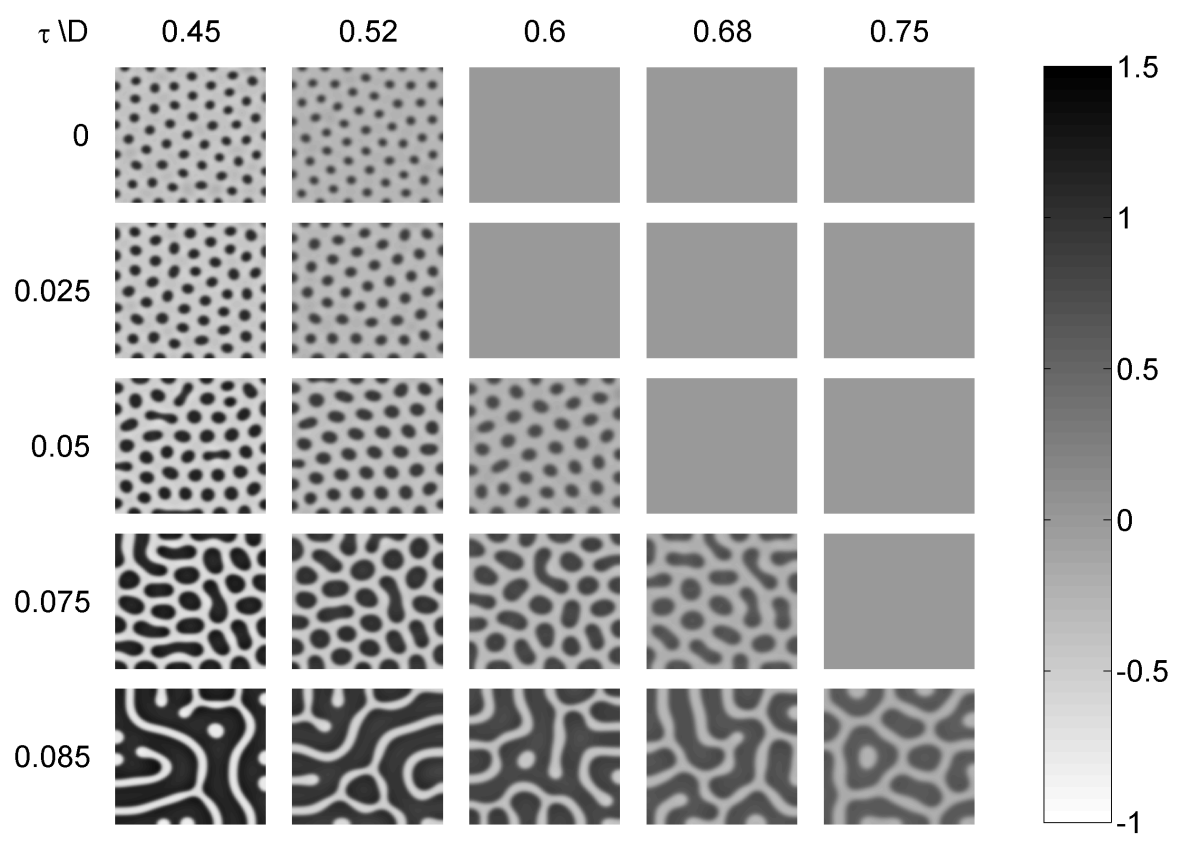

Figure 5. Dependence of patterns on the ratio of diffusions $D$ and the strength of the unilateral source $\tau$ for the nonsmooth unilateral term $\hat{g}(v)=\tau v^{-}$. Each box corresponds to the indicated values of $D$ and $\tau$ and to parameter values (4).

as in the case of system (3) with $\hat{g}(v)=-\tau v$. Although, we do not present the results, we solved system (3) with the nonsmooth unilateral term many times for values $D$ close to the critical one and all these results confirmed this hypothesis.

On the other hand, comparison of Figures 5 and 6 clearly reveals the difference of the resulting patterns. The difference is even qualitative. While the patterns produced by the unilateral term are irregular with large irregular spots, patterns produced by the linear term are approximately symmetric with smaller circular spots. This qualitative difference can be explained by the substantial difference of the corresponding nonlinear dynamics especially for values of $v$ distant from the ground state.

Another difference can be visible if we observe how the patterns change as $\tau$ is increased. In both cases the spots grow bigger, but differently. For the unilateral term the spots grow, fill gaps among them, and finally merge. On the other hand, for the linear term the distances of spots grow proportionally and spots do not merge. This behaviour is similar as if we scaled the system by the size of the domain $\Omega$ or by parameter $\delta$. We note that in the dimensionless system [1] 


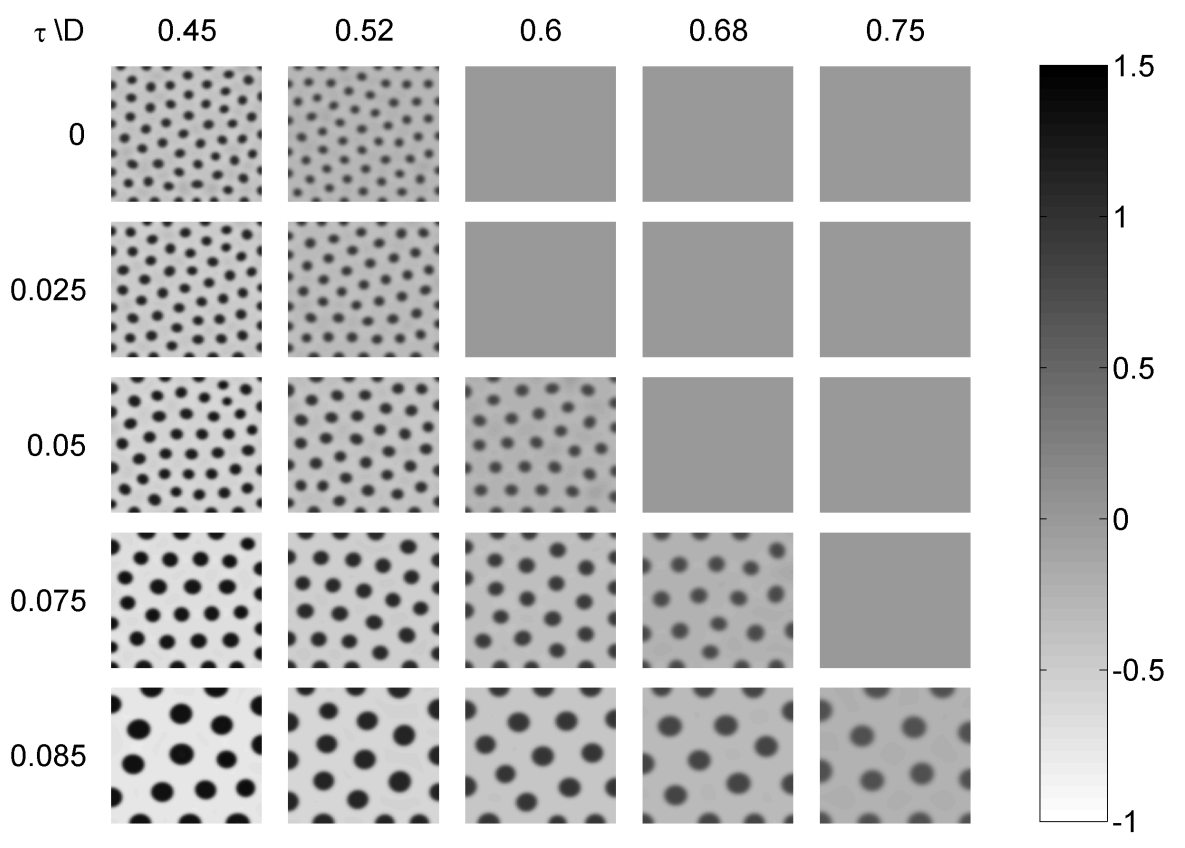

Figure 6. Dependence of patterns on $D$ and $\tau$ for the choice $\hat{g}(v)=-\tau v$. Each box corresponds to the indicated values of $D$ and $\tau$ and to parameter values (4).

(where no unilateral term is considered) a parameter $\eta$ scales the system in the same manner.

\subsection{Unilateral source as a model of a receptor-based morphogen reg-}

ulation. It is usually considered in reaction-diffusion models of pre-pattern formation in mammalian skin that morphogens are proteins (ligands) secreted to the extracellular space [19]. These proteins do not react directly with each other but they bind to cell membrane receptors and the production of morphogens is subsequently regulated by signaling pathways. The mechanism of cell response to morphogen gradients is a subject of intensive debates [35, 2]. It is assumed here that the number of receptors engaged with ligands influences the rate of

\begin{tabular}{c|cccccc}
$\tau$ & 0 & 0.025 & 0.05 & 0.075 & 0.08 & 0.085 \\
\hline$D_{\text {crit }}$ & 0.53 & 0.57 & 0.62 & 0.71 & 0.74 & 0.78
\end{tabular}

TABLE 1. Critical ratios (10) of diffusion coefficients for the linear source term (i.e. system (3) with $\hat{g}(v)=-\tau v$ ) and various values of $\tau$. Rounded to two significant digits. 
morphogen production [18 and that this influence is in inverse proportion. The introduction of a unilateral term to our model reflects a limited number of receptors in a membrane: if the concentration of a morphogen exceeds the threshold value $\theta$, all receptors are occupied independently on the amount how much the threshold is exceeded. The system is saturated and does not produce the corresponding morphogen. In the case of the morphogen $v$, this process is well described by the term $\hat{g}(v)=\tau(v-\theta)^{-}$. Indeed, in points $(x, y) \in \Omega$ and times $t$, where $v(x, y, t)<\theta$, the term $\tau(v(x, y, t)-\theta)^{-}$is positive and works as a source term in (1). On the other hand, in points $(x, y) \in \Omega$ and times $t$, where $v(x, y, t) \geq \theta$, the term $\tau(v(x, y, t)-\theta)^{-}$vanishes and has no effect.

3.5. Case study: Coat patterns of cheetahs. It has been shown that Taqpep gene is responsible for the regularity of pre-pattern in the case of domestic cats and cheetahs [22], see Figure 7 (left). King cheetahs have a mutation in this gene and their specific coat pattern is characterized by irregular, large spots, see Figure 7 (right). Taqpep encodes a type II membrane-spanning protein of the M1 aminopeptidase family whose metalloprotease-containing ectodomain (further denoted as MCE) can diffuse outside the cell.

It has been proposed that a reaction-diffusion model is suitable to elucidate a role of MCE for the constitution of pre-patterns [21. Mathematical model (3) and considerations from Section 3.4 serves this purpose, presuming that variable $v$ is the deviation of the MCE concentration in the extracellular space from its equilibrium concentration $\bar{V}$.

According to the model, the unilateral regulation is weak in the common morph of cheetah ( $\tau$ close to zero), resulting in the usual spotted pattern, whereas the mutation in Taqpep gene yields stronger unilateral regulation in the case of the king cheetah ( $\tau$ around 0.08 ). The simulated patterns relate to real skin patterns, which applies both for the common morph of cheetah, see Figures 4 (middle) and 7 (left), and the king cheetah, see Figures 4 (left) and 7 (right).

\section{DisCUSSION AND CONCLUSIONS}

In this contribution we investigated a reaction-diffusion system with a nonsmooth unilateral source term $\hat{g}(v)=\tau v^{-}$and its approximations. We provided a case study for a particular system and analysed numerically the influence of such source term on the Turing instability and on the resulting patterns. We explained a possible biological meaning of this term and obtained the following conslusions.

Sensitivity of the Turing instability. The linear analysis of systems with smooth approximations of the term $\hat{g}(v)=\tau v^{-}$is not informative about the Turing instability of the system with the nonsmooth term. Small perturbations of the ground state can evolve to patterns for one approximation of the nonsmooth term, but not for the other even though they are arbitrarily accurate. This shows that the Turing instability is sensitive to small changes of the nonlinear dynamics. 

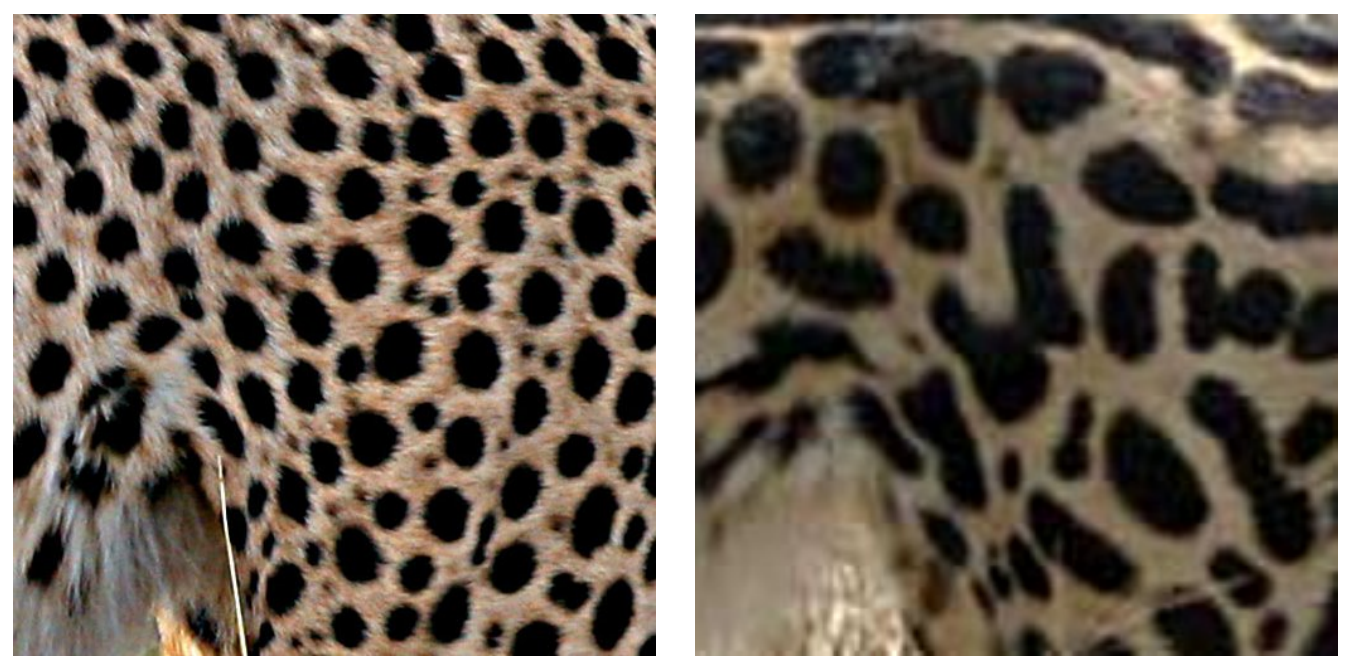

FiguRE 7. Typical coat patterns of the common morph of the cheetah (left) and king cheetah (right).

Robustness of Turing patterns. We also showed that initial perturbations of the ground state larger than a certain minimal size do robustly evolve to patterns for both the nonsmooth term and its approximations. In addition, these patterns are almost identical regardless the particular form of the unilateral term in the small neighbourhood of the ground state.

Irregularity of patterns. We have found that unilateral sources break the approximate regularity and symmetry of the usual patterns. System (3) with the unilateral term $\hat{g}(v)=\tau v^{-}$produces spots with irregular shapes and variable distances between them. This contrasts to the classical smooth systems corresponding to choices $\hat{g} \equiv 0$ and $\hat{g}(v)=-\tau v$ in (3), where we observe closeto-regular disc-shaped spots approximately symmetrically placed, see Figure 4. Thus, the unilateral sources prescribed for the inhibitor $v$ break the regularity of patterns for all values of diffusion constants yielding patterns, provided the strength of the unilateral source is not negligible.

Patterns for higher ratio of diffusion parameters. Interestingly, system (3) with the unilateral term $\hat{g}(v)=\tau v^{-}$produces patterns even for those values of diffusion constants which prevent any pattern formation in the original system (i.e. $\hat{g} \equiv 0$ ). Further, we observe that the critical ratio of diffusions for the system with $\hat{g}(v)=\tau v^{-}$seems to be identical to the critical ratio of the system with $\hat{g}(v)=-\tau v$. However, the resulting patterns differ considerably as we mentioned in the previous paragraph.

We verified these conclusions numerically for the particular system (3), but we believe that they are valid for other kinetics as well. We verified this conclusion in 34 for a unilateral term added to the Thomas model [37. This indicates that our findings about the effects of the unilateral term are not limited to a 
single kinetics. The generality of these results is also supported by earlier results [10, 23, 27], which guarantee the existence of bifurcations of spatial patterns and certain instability of the ground state for large $d_{1} / d_{2}$ for a unilateral source described by variational inequalities, but they are valid for a very large class of kinetics.

Reaction-diffusion systems with nonsmooth nonlinear unilateral terms are interesting from both the theoretical and practical points of view. In contrasts to the classical smooth case, where the small perturbations initially evolve according to a linear dynamics, the evolution of small perturbations of the ground state for the nonsmooth unilateral term is inherently governed by a nonlinear dynamics. This nonlinear dynamics may yield completely new phenomena in the pattern formation mechanisms. In this contribution, we have made an attempt towards the understanding of the unilateral terms in models of biological patterns formation. However, further research is necessary for the investigation of feasible biological applications.

\section{ACKNOWLEDGMENTS}

The research leading to these results has received funding from the People Programme (Marie Curie Actions) of the European Union's Seventh Framework Programme (FP7/2007-2013) under REA grant agreement no. 328008. Further, M.K. has been supported by the Grant 13-00863S of the Czech Science Foundation and T.V., M.K., and V.R. acknowledge the support of RVO 67985840.

\section{REFERENCES}

[1] Aragón, J., Barrio, R., Woolley, T., Baker, R., Maini, P.: Nonlinear effects on Turing patterns: Time oscillations and chaos. Physical Review E 86(2), 026,201 (2012)

[2] Ashe, H.L., Briscoe, J.: The interpretation of morphogen gradients. Development 133(3), 385-394 (2006)

[3] Asslani, M., Di Patti, F., Fanelli, D.: Stochastic turing patterns on a network. Physical Review E 86(4), 046,105 (2012)

[4] Barrio, R., Varea, C., Aragón, J., Maini, P.: A two-dimensional numerical study of spatial pattern formation in interacting turing systems. Bulletin of mathematical biology 61(3), 483-505 (1999)

[5] Biancalani, T., Fanelli, D., Di Patti, F.: Stochastic turing patterns in the brusselator model. Physical Review E 81(4), 046,215 (2010)

[6] Biancalani, T., Jafarpour, F., Goldenfeld, N.: Giant amplification of noise in fluctuationinduced pattern formation. Physical review letters 118(1), 018,101 (2017)

[7] Cantini, L., Cianci, C., Fanelli, D., Massi, E., Barletti, L., Asllani, M.: Stochastic amplification of spatial modes in a system with one diffusing species. Journal of mathematical biology 69(6-7), 1585-1608 (2014)

[8] Chen, Y., Schier, A.F.: The zebrafish nodal signal squint functions as a morphogen. Nature 411(6837), 607-610 (2001)

[9] Ciarlet, P.G.: The finite element method for elliptic problems. North-Holland Publishing Co., Amsterdam-New York-Oxford (1978). Studies in Mathematics and its Applications, Vol. 4 
[10] Drábek, P., Kučera, M., Míková, M.: Bifurcation points of reaction-diffusion systems with unilateral conditions. Czechoslovak Mathematical Journal 35(4), 639-660 (1985)

[11] Economou, A.D., Ohazama, A., Porntaveetus, T., Sharpe, P.T., Kondo, S., Basson, M.A., Gritli-Linde, A., Cobourne, M.T., Green, J.B.: Periodic stripe formation by a Turing mechanism operating at growth zones in the mammalian palate. Nature genetics $\mathbf{4 4}(3)$, 348-351 (2012)

[12] Edelstein-Keshet, L.: Mathematical models in biology. McGraw-Hill, Boston (1988)

[13] Eisner, J., Kučera, M.: Spatial patterning in reaction-diffusion systems with nonstandard boundary conditions. Fields Inst. Commun. 25, 239-256 (2000)

[14] Eisner, J., Kučera, M.: Bifurcation of solutions to reaction-diffusion systems with jumping nonlinearities. In: A. Sequeira, H. Beirao da Veiga, J.H. Videman (eds.) Applied Nonlinear Analysis, pp. 79-96. Springer (2002)

[15] Eisner, J., Väth, M.: Location of bifurcation points for a reaction-diffusion system with Neumann-Signorini conditions. Advanced Nonlinear Studies 11(4), 809-836 (2011)

[16] Fanelli, D., Cianci, C., Di Patti, F.: Turing instabilities in reaction-diffusion systems with cross diffusion. The European Physical Journal B 86(4), 142 (2013)

[17] Filippov, A.: Differential Equations with Discontinuous Righthand Sides. Kluwer Academic Publishers (1988)

[18] Gurdon, J., Bourillot, P.Y.: Morphogen gradient interpretation. Nature 413(6858), 797$803(2001)$

[19] Headon, D.J., Painter, K.J.: Stippling the skin: Generation of anatomical periodicity by reaction-diffusion mechanisms. Mathematical Modelling of Natural Phenomena 4(4), 83-102 (2009)

[20] Jones, D.S., Sleeman, B.D.: Differential Equations and Mathematical Biology. Chapman \& Hall (2003)

[21] Kaelin, C.B., Barsh, G.S.: Genetics of pigmentation in dogs and cats. Annu. Rev. Anim. Biosci. 1(1), 125-156 (2013)

[22] Kaelin, C.B., Xu, X., Hong, L.Z., David, V.A., McGowan, K.A., Schmidt-Küntzel, A., Roelke, M.E., Pino, J., Pontius, J., Cooper, G.M., et al.: Specifying and sustaining pigmentation patterns in domestic and wild cats. Science 337(6101), 1536-1541 (2012)

[23] Kim, I.S., Väth, M.: The Krasnoselskii-Quittner formula and instability of a reactiondiffusion system with unilateral obstacles. Dynamics of Partial Differential Equations 11(3), 229-250 (2014)

[24] Klika, V., Baker, R.E., Headon, D., Gaffney, E.A.: The influence of receptor-mediated interactions on reaction-diffusion mechanisms of cellular self-organisation. Bulletin of mathematical biology 74(4), 935-957 (2012)

[25] Kondo, S.: The reaction-diffusion system: a mechanism for autonomous pattern formation in the animal skin. Genes to Cells 7(6), 535-541 (2002)

[26] Kondo, S., Miura, T.: Reaction-diffusion model as a framework for understanding biological pattern formation. Science 329(5999), 1616-1620 (2010)

[27] Kučera, M., Väth, M.: Bifurcation for a reaction-diffusion system with unilateral and Neumann boundary conditions. Journal of Differential Equations 252(4), 2951-2982 (2012)

[28] Kůs, P.: Convergence and stability of higher-order finite element solution of reactiondiffusion equation with turing instability. In: J. Brandts, S. Korotov, M. Křížek, K. Segeth, J. Šístek, T. Vejchodský (eds.) Application of Mathematics 2015, pp. 140-147. Institute of Mathematics CAS (2015)

[29] Kučera, M., Bosák, M.: Bifurcation for quasi-variational inequalities of reaction-diffusion type. Stability and Applied Analysis of Continuous Media 3(2), 354-369 (1994)

[30] Liu, R., Liaw, S., Maini, P.: Two-stage Turing model for generating pigment patterns on the leopard and the jaguar. Physical review E 74(1), 011914 (2006) 
[31] Mou, C., Jackson, B., Schneider, P., Overbeek, P.A., Headon, D.J.: Generation of the primary hair follicle pattern. Proceedings of the National Academy of Sciences 103(24), 9075-9080 (2006)

[32] Murray, J.D.: Mathematical biology. II. Spatial models and biomedical applications. Springer-Verlag (2003)

[33] Nishiura, Y.: Global structure of bifurcating solutions of some reaction-diffusion systems. SIAM Journal on Mathematical Analysis 13(4), 555-593 (1982)

[34] Rybár, V., Vejchodský, T.: Irregularity of Turing patterns in the Thomas model with a unilateral term. In: J. Chleboun, P. Přikryl, K. Segeth, J. Š́stek, T. Vejchodský (eds.) Programs and Algorithms of Numerical Matematics 17, pp. 188-193. Institute of Mathematics AS CR (2015)

[35] Sagner, A., Briscoe, J.: Morphogen interpretation: concentration, time, competence, and signaling dynamics. Wiley Interdisciplinary Reviews: Developmental Biology 6, e271 (2017). DOI 10.1002/wdev.271

[36] Schier, A.F.: Nodal morphogens. Cold Spring Harbor perspectives in biology 1(5), a003459 (2009)

[37] Thomas, D.: Artificial enzyme membranes, transport, memory, and oscillatory phenomena. In: D. Thomas, J.P. Kernevez (eds.) Analysis and control of immobilized enzyme systems, pp. 115-150. North Holland (1976)

[38] Turing, A.M.: The chemical basis of morphogenesis. Philosophical Transactions of the Royal Society of London B: Biological Sciences 237(641), 37-72 (1952)

[39] Woolley, T.E., Baker, R.E., Maini, P.K., Aragón, J.L., Barrio, R.A.: Analysis of stationary droplets in a generic turing reaction-diffusion system. Physical Review E 82(5), 051,929 (2010)

Tomáš Vejchodský, Institute of Mathematics, Academy of Sciences, Žitná 25, CZ-115 67 Praha 1, Czech Republic,

E-mail address: vejchod@math.cas.cz

Filip Jaroš, Faculty of Arts, University of Hradec Králové, náměstí Svobody 331, CZ-500 02 Hradec Králové, Czech Republic.

E-mail address: filip.jaros@uhk.cz

Milan Kučera, Institute of Mathematics, Academy of Sciences, Žitná 25, CZ11567 Praha 1, Czech Republic and Department of Mathematics, Faculty of Applied Sciences, University of West Bohemia in Pilsen, Univerzitní 8, 30614 PlzeŇ, Czech Republic.

E-mail address: kucera@math.cas.cz

Vojtěch RybáŘ, Institute of Mathematics, Academy of Sciences, Žitná 25, CZ11567 Praha 1, Czech Republic.

E-mail address: rybar@math.cas.cz 